\title{
Primary hydatid disease of the occipital bone presenting as space occupying cystic mass of the posterior fossa
}

\author{
Erkin Özgiray*a, Melih Bozkurt ${ }^{\mathrm{b}}$, Oğuz Resat Sipahic, Uğur Oğula, Tayfun Dalbastı ${ }^{\mathrm{a}}$ \\ ${ }^{a}$ Department of Neurosurgery, Medical Faculty, Ege University, Izmir, Turkey \\ ${ }^{b}$ Department of Neurological Surgery, Medical Faculty and Public Health, University of Wisconsin, Madison, Wisconsin, USA \\ 'Department of Infectious Diseases and Clinical Microbiology, Medical Faculty, Ege University, Izmir, Turkey
}

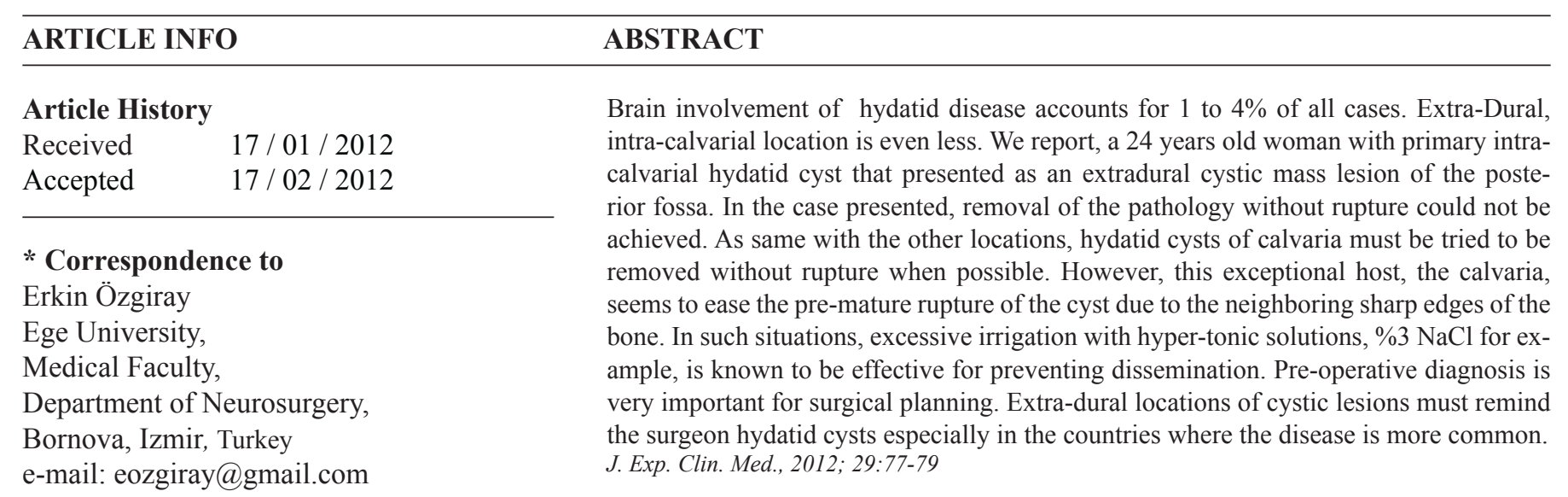

\section{Keywords:}

Echinococcus granulosus

Hydatid disease

Calvarium

Cyst hydatid

Posterior fossa

C 2012 OMU All rights reserved

\section{Introduction}

Hydatid disease is a worldwide zoonozis. Echinococcus granulosus and Echinococcus multilocularis causes the two common types of hydatid disease, cystic and alveolar echinococcosis respectively (McManus et al., 2003; Beskonakli et al., 2005). Unlike E. granulosus which produces cystic lesions, E. multilocularis produces invasive solid lesions, full of connective tissue and a jelly-like material (Oktar et al., 1999). Although hydatid cyst of E. granulosus may indwell in any organ of the human body, the most affected ones are liver and lung (McManus et al., 2003; Beskonakli et al., 2005).

Cerebral involvement of hydatid disease is reported only up to $4 \%$ of cases (Ersahin et al., 1993; Beskonakli et al., 1996; Erman et al., 2001; Beskonakli et al., 2005). It accounts for $0.02 \%$ to $0.22 \%$ of all intracranial space-occupying lesions (Furtado et al., 2009). Beskonakli et al. (2005) classifies the intracranial hydatid disease in three forms which are consisted of intracerebral, extra-cerebral and combined. In this paper we report, a 24 years old woman with primary intracalvarial hydatid cyst which fulfills the pure extra-cerebral form. In the case presented, removal of the pathology without rupture could not be achieved.

\section{Case}

Twenty four-year-old woman was admitted to a physician with the complaints of insisting headache and newly onset of dizziness. When the cranial Magnetic Resonanse Imaging (MRI) taken on the order of the first physician revealed a cystic mass of posterior fossa, the patient was immediately referred to our department. On the admission her neurological examination revealed detoriated cerebellar tests with a mild trunckal ataxia. Papillary stasis was seen on fundoscopic examination bilaterally. Besides these pathological findings, patient was found to be fully conscious with a score of 15 in Glasgow Coma Scale (GCS). Her headache was prominent especially in the mornings with a new onset of dizziness. 
Cranial pre-and post-contrast MRI suggested an extra-axial location of a large cystic mass with fine contours (Fig. 1a, 1b).

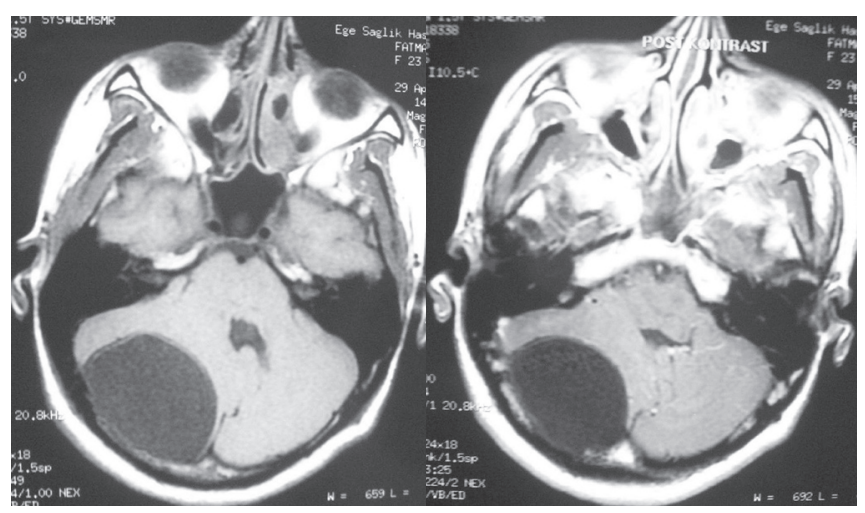

Fig. 1. T1 weighted pre- (a) and post- contrast (b) axial MR images depicting fine contoured extra-axial cystic mass lesion in the posterior fossa on right cerebellar cortex. Cystic walls are faintly enhanced after gadolinium administration (b).

An operation was carried out on a routine scheduled day for total resection of the cystic mass. Head was put on a threepin holder and the patient was positioned prone. A right paramedian sub-occipital approach was planned. Soon after the skin incision the exposed occipital bone depicted an area of erosion about $3 \times 3 \mathrm{~cm}$ with a membrane shining under tension through the bone deficit. Despite the extreme attention for keeping the cyst un-ruptured, during the excision of the bone flap, membrane was punctured. Content of the cyst with its membrane defined the pathology as hydatid cyst. All the mass was located at extra-dural space that originating from the occipital bone. Immediately after diagnosing the disease, excessive irrigation with hypertonic saline was performed. Meanwhile albendazole was made ready in the operation room and finally it was used for extra-dural irrigation. Rest of the operation was performed in regular fashion with bleeding control and closure of the layers properly. Patient was discharged after three more days of hospitalization with an uneventful recovery. She was started albendazole $400 \mathrm{mg} / \mathrm{q}$ $12 \mathrm{~h}$ per oral before discharge and her further medication and follow-up was planned with collaboration of the infectious disease department.

\section{Discussion}

Infestation with the tapeworm E. granulosus and less frequently, E. multilocularis causes echinococcosis (hydatidosis) (Ciurea et al., 2006). The disease is reported to be endemic in South America, Australia, India, northwest China, Mediterranean and the Middle East (Ersahin et al., 1993; McManus et al., 2003; Ciurea et al., 2006). But it is possible that it may be seen more often in the western countries in near future because of increasing rates of migration and travelling. The incidence of the disease ranges from 3 to 50 cases per 100,000 inhabitants in endemic areas (Krajewski and Stelmasiak, 1991; Ciurea et al., 2006). Highest rates are among the communities involved in sheep farming where the sheep strain of E. granulosus in transmission to people (McManus et al., 2003). Lungs and liver are the most common sites for the hydatid cysts and cranial manifestation is rare and accounts less then 4\% of all cases (Ersahin et al., 1993; McManus et al., 2003; Ciurea et al., 2006; Furtado et al., 2009; Is et al.,
2009). When cysts occur intracranially, they are usually unilocular. They prefer intracerebral and supratentorial localization in the distribution of the terminal branches of the middle cerebral artery, usually temporal and parietal lobes (Ersahin et al., 1993; Erman et al., 2001; Ciurea et 1., 2006; Kayaoglu, 2008; Is et al., 2009). Hydatid cysts are scarcely located both in the calvaria and the posterior fossa (Erman et al., 2001; Polat et al., 2003; Kayaoglu, 2008; Is et al., 2009). Only a few extradural posterior fossa cysts are reported in the English literature (Beskonakli et al., 1996; Erman et al., 2001).

The initial phase of the primary infection is always asymptomatic. Small cysts may remain asymptomatic for many years. The incubation period of cystic echnococcosis may vary from many months to years and is not known clearly. The infection may be manifest by rupture of the cysts or the mass effect (McMnaus et al., 2003). But cerebral location of the disease probably causes an earlier onset as 50 to $75 \%$ of cerebral hydatid cysts occur in childhood (Ersahin et al., 1993; Is et al., 2009).

Although radiological investigations are very helpful in identifying hydatid cysts preoperatively the certain diagnosis remains to be a pathological one. Computerized Tomography (CT) and MRI are the current diagnostic modalities of choice. MRI appears to be superior to CT in the detection of rim enhancement and surrounding edema in the brain parenchyma (Ciurea et al., 2006). The role of diffusion weighted MRI in the diagnosis of cerebral hydatid disease is currently under investigation (Kitis et al., 2004; Ciurea et al., 2006). According to Kitis et al. (2004) cerebral E. granulosus lesions, unless complicated, reveal CSF-like MR signal intensity on diffusion weighted intensity. CT is advantageous in rare cases of hydatid cysts located at the calvaria. It is expected to depict the classical findings of intraosseous solitary cystic lesion with regular contours (Erman et al., 2001). Lack of pre-operative CT scan probably diminished the chance of pre-diagnose in our case. But its existence naturally do not guarantee the exact diagnose prior to the operation as eosinophilic granuloma, cystic fibrous dysplasia and calvarial epidermoid tumour must be considered in the differential diagnosis of calvarial hydatid cyst (Erman et al., 2001).

Certain treatment of hydatid cyst is total resection avoiding rupture (Lunardi et al., 1991; Ersahin et al., 1993; Erman et al., 2001). Dowling has defined and named the technique himself, as giving birth to the intracerebrally located cysts by continuous irrigation with hypertonic saline between the cyst and the brain tissue (Carrea et al., 1975). But in rare localization of calvaria, no definite techniques are defined. Principally a large craniectomy surrounding the eroded center of the calvarial bone sounds to be effective whilst in practice all the cases are reported to be early ruptured before the excision. Early rupture may be a resultant of sharp bony edges surrounding the defect eroded by the cyst and its adherence to the dura and osseous trabeculae. In case of early rupture intraoperatively, extensive irrigation of the site with a hypertonic solution is strongly recommended (Erman et al., 2001).

Obstructive hydrocephalus and subdural effusions are well known post-operative complications (Beskonakli et al., 2005; Ciurea et al., 2006). In one of the recent and largest series, Ciurea et al., (2006) report a rate of $36.8 \%$ for postoperative subdural effusion. Seizures, porencephalic cysts and non-bacterial meningitis are among other post-operative 
complications reported in the literature (Lunardi et al., 1991; Beskonakli et al., 2005; Ciurea et al., 2006). Rupture of the cyst during surgery may cause anaphylaxis and mortality in the per-operative period (Ersahin et al., 1993; McManus et al., 2003). Our case was discharged after an uneventful recovery and early post-operative CT-scan revealed no pathologies secondary to the operation.

We conclude that rare phenomenon of intra calvarial and extra-dural hydatid cysts must be kept among the differential diagnosis of cystic masses of this location. Although un-ruptured excision of these cysts is primary goal, this unique location surrounded by sharp bony edges seems to be obstructing this aim. In case of pre-mature rupture, excessive irrigation with hypertonic saline and albendazole is recommended. Anti-echnicoccal medical therapy must be continued postoperatively.

\section{REFERENCES}

Beskonakli, E., Solaroglu, I., Tun, K., Albayrak, L., 2005. Primary intracranial hydatid cyst in the interpeduncular cistern. Acta. Neurochir. 147, 781-783.

Beşkonakli, E., Cayli, S., Yalçinlar, Y., 1996. Primary intracranial extradural hydatid cyst extending above and below the tentorium. Br. J. Neurosurg. 10, 315-316.

Carrea, R., Dowling, E. Jr., Guevara, A., 1975. Surgical treatment of hydatid cysts of the central nervous system in the pediatric age (Dowling's technique). Child's Brain. 1, 4-21.

Ciurea, A.V., Fountas, K.N., Coman, T.C., Machinis, T.G., Kapsalaki, E.Z., Fezoulidis, N.I., Robinson, J.S., 2006. Long-term surgical outcome in patients with intracranial hydatid cyst. Acta. Neurochir. 148, 421-426.

Erman, T., Tuna, M., Göçer, I., Ildan, F., Zeren, M., Cetinalp, E., 2001. Intracranial intraosseous hydatid cyst. Case report and review of literature. Neurosurg. Focus. 11, ECP1.

Erşahin, Y., Mutluer, S., Güzelbağ, E., 1993. Intracranial hydatid cysts in children. Neurosurgery. 33, 219-224.

Furtado, S.V., Visvanathan, K., Nandita, G., Reddy, K., Hegde, A.S., 2009. Multiple fourth ventricular hydatidosis. J. Clin. Neurosci. 16, 110112.

Is, M., Gezen, F., Akyuz, F., Aytekin, H., Dosoglu, M., 2009. A 13-year-old girl with a cystic cerebellar lesion: Consider the hydatid cyst. J. Clin. Neurosci. 16, 712-713.

Kayaoglu, C.R., 2008. Giant hydatid cyst in the posterior fossa of a child: A case report. J. Int. Med. Res. 36, 198-202.

Krajewski, R., Stelmasiak, Z., 1991. Cerebral hydatid cysts in children. Childs Nerv. Syst. 7, 154-155.

Kitis, O., Calli, C., Yunten, N., 2004. Report of Diffusion-Weighted MRI in two cases with different cerebral hydatid disease. Acta. Radiol. 45, $85-87$.

Lunardi, P., Missori, P., Lorenzo, N.D., Fortuna, A., 1991. Cerebral hydatidosis in childhood: A retrospective survey with emphasis on long-term follow-up. Neurosurg. 29, 515-518.

McManus, D.P., Zhang, W., Li J., Bartley, P.B., 2003. Echinococcosis. Lancet. 362, 1295-1304.

Oktar, N., Karabıyıkoğlu, M., Demirtaş, E., Altıntaş, N., Korkmaz, M., Özdamar, N., 1999. Cerebral alveolar Echinococcosis. Review of the literature and report of a case. J.Neurol.Sci.Turk. 161-164.

Polat, P., Kantarci, M., Alper, F., Suma, S., Koruyucu, M.B., Okur, A., 2003. Hydatid disease from head to toe. Radiographics. 23, $475-494$. 